\title{
Transneuronal spread of alpha herpesvirus infection: lessons learned from using PRV as a neural circuit tracer Lynn W Enquist
}

Address: Department of Molecular Biology, Princeton University, Princeton, New Jersey, 08544, USA

Email: Lynn W Enquist - Lenquist@princeton.edu

from Infectious diseases of the nervous system: pathogenesis and worldwide impact

Paris, France. 10-13 September 2008

Published: 23 September 2008

BMC Proceedings 2008, 2(Suppl I):SI4

This abstract is available from: http://www.biomedcentral.com/I753-656I/2/SI/SI4

(c) 2008 Enquist; licensee BioMed Central Ltd.

The alpha herpesviruses have evolved to enter the peripheral nervous systems of their natural hosts and establish a quiescent infection (latency) in peripheral ganglia that can be reactivated at a later time. The spread of infection is through chains of synaptically connected neurons. The biology of pseudorabies virus (PRV), a swine herpesvirus with a very broad host range (essentially all mammals except higher primates), is of some interest for several reasons. In all of its hosts except swine, PRV infection is neuroinvasive and lethal. In addition, certain attenuated mutants can spread only in one direction in chains of synaptically connected neurons. These strains are used in tracing neural circuitry in a variety of mammals and bird embryos. The viral genome inside capsids is moved long distances in axons in a controlled fashion on microtubules. Sorting of virion components into axons controls the directional, neuronal spread of infection of alpha herpesviruses. We have been studying two aspects of the general problem. First, using GFP and mRFP fusion proteins, we have used video confocal microscopy to assess mechanisms and gene products that influence spread of Pseudorabies (PRV) virion components within axons. Second, we have developed an in vitro cell culture system that enables us for the first time, to study axon-mediated, transneuronal spread from neurons to neurons as well as neuron to non-neuronal cells similar to what happens after reactivation and spread to epithelial surfaces. These systems appear to duplicate all the known in vivo correlates of transneuronal spread and provide the opportunity to do both quantitative and qualitative assessment of spread of PRV infection.

\author{
Funding \\ NIH RO133506, NCRR P40 RRO1 18604
}

